\title{
Sclerosing mesenteritis:an uncommon cause of chronic abdominal pain
}

\begin{abstract}
Sclerosing mesenteritis (SM), also known as mesenteric panniculitis or mesenteric lipodystrophy, is a rare disease that affects the mesentery of the small intestine. We present a case of 59 years old with chronic abdominal pain and incarcerated umbilical hernia diagnosed as SM by CT scan which was confirmed by histopathology. Patient continued to have chronic low grade abdominal pain even after correction of the hernia. Repeat CT scan continued to show stable SM. SM is usually associated with underlying conditions such as lymphoma, peritoneal carcinomatosis, mesenteric fibromatosis, mesenteric edema and carcinoid tumor which needs to be ruled out. SM usually follows a benign and slowly progressive course. Asymptomatic patients typically require no treatment, while refractory cases have shown some symptomatic improvement with immunosuppressive therapy.
\end{abstract}

Keywords: histopathology, immunosuppressive therapy, mesenteric fibromatosis, peritoneal carcinomatosis, mesenteric lipodystrophy
Volume 9 Issue 3 - 2018

\author{
Santosh Raj Sharma,' Denzil Etienne,' Dhuha \\ Alhankawi, ${ }^{2}$ Jaafar Al-dahwi, 'Madhavi Reddy' \\ 'The Brooklyn Hospital Center,Academic Affiliate of The Icahn \\ School of Medicine at Mount Sinai, USA \\ ${ }^{2}$ Department of Medicine, New York University Langone \\ Medical Center, USA
}

\begin{abstract}
Correspondence: Dhuha Alhankawi, New York University langone Medical Center, Department of Medicine, Division of Gastroenterology, New York, NY, USA,

Email Dhuha.Alhankawi@nyumc.org
\end{abstract}

Received: April 09, 2018 | Published: June II, 2018
Abbreviations: SM, sclerosing mesenteritis; $\mathrm{CBC}$, complete blood count; BMP, basic metabolic panel; LFT, liver function tests; CRP, C-reactive protein; ESR, erythrocyte sedimentation rate

\section{Introduction}

The mesentery is formed as a fold of the peritoneum that supports the intestine to the abdominal wall which contains numerous structures like blood vessels, nerves and the lymphatic system. Sclerosing mesenteritis (SM) is a rare disease of the mesentery characterized by chronic inflammation and fibrosis of the mesentery which was first described by Jura as "retractile mesenteritis" in $1924^{1}$ and the term sclerosing mesenteritis was given by Emory et al. ${ }^{2}$ Only around 1200 cases have been reported ${ }^{3}$ however, the prevalence is thought to be around $0.6 \%$ and many cases remain undiagnosed. ${ }^{4} \mathrm{SM}$ is known by various names some of which are Feiffer-Weber-Christian disease, Xanthogranulomatous mesenteritis, mesenteric lipodystrophy, retractile mesenteritis, sclerosing mesenteritis, liposclerotic mesenteritis, lipomatosis and lipogranuloma of the mesentery. ${ }^{1} \mathrm{We}$ present a rare case of SM in a 50years old male who presented with abdominal pain.

\section{Case presentation}

A 58-year-old white male with a past medical history of coronary artery disease, gastroesophageal reflux disease, hyperlipidemia and partially incarcerated umbilical hernia was referred to the surgeon by his primary care physician due to chronic right lower quadrant abdominal pain associated with one episode of non-bloody vomiting. He denied change in bowel habits, weight loss or blood in stools. On physical examination the only positive finding was that the abdomen was soft with mild tenderness in the right low quadrant and no peritoneal signs. Complete Blood Count (CBC), Basic Metabolic Panel (BMP) and Liver Function Tests (LFT) were essentially within normal limits. The total cholesterol was $316 \mathrm{mg} / \mathrm{dl}$, high density lipid was $38 \mathrm{mg} / \mathrm{dl}$ and triglycerides level were $203 \mathrm{mg} / \mathrm{dl}$. Lactic acid, Erythrocyte Sedimentation Rate (ESR) and C-Reactive Protein (CRP) were also normal. CT abdomen/pelvis with contrast revealed increased mesenteric attenuation with induration and associated lymphadenopathy within the inferior midline abdomen (Figure 1). Lymph nodes demonstrated a halo of spared fat (Figure 2) consistent with sclerosing mesenteritis. The patient subsequently underwent elective laparoscopic epigastric and umbilical herniorrhaphy with mesh. The surgical specimen pathology consisted of adipose tissue. The histologic finding was mesothelial lined fibrous tissue showing chronic inflammation. It negative for malignancy including lymphoma. Upper Gastrointestinal Endoscopy (EGD) was performed and was negative for malignancy and Helicobacter Pylori. Patient was discharged from the hospital without any complications with pain medications. On subsequent follow up, the patient continued to complain of chronic mild right lower abdominal pain that was controlled with non-steroidal anti-inflammatory drugs . Repeat CT abdomen/pelvis one year later showed stable appearance of inflamed fat at the root of mesentery with numerous small lymph nodes (Figure 3).

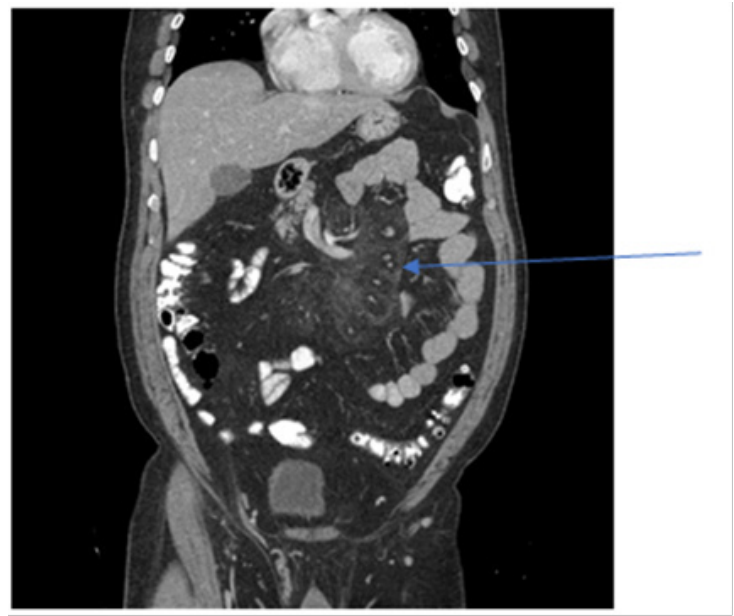

Figure I Mesenteric attenuation with induration and associated lymphadenopathy within the inferior midline abdomen. 


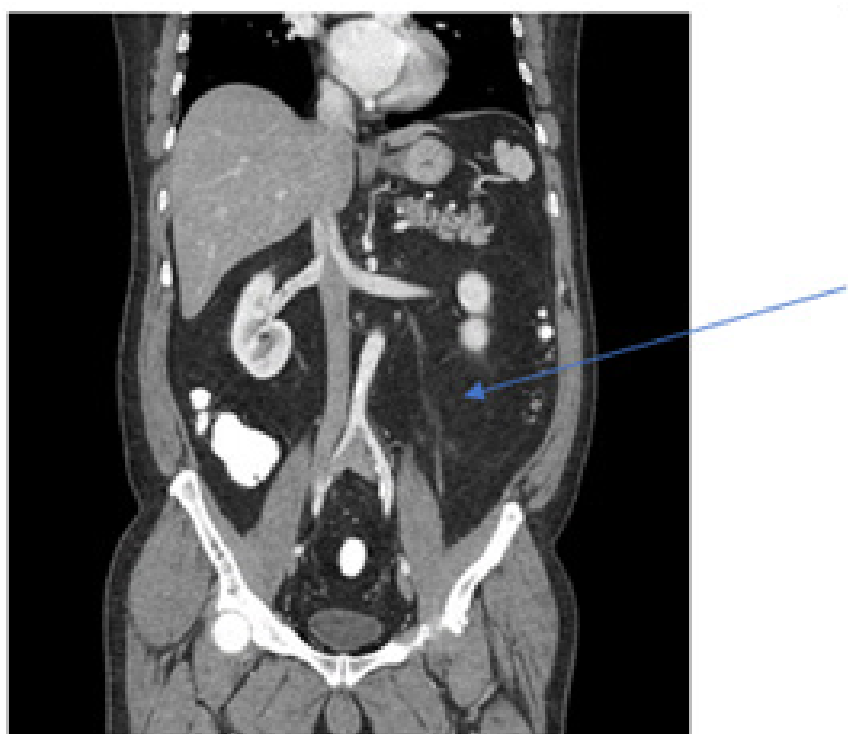

Figure $\mathbf{2}$ Lymph nodes demonstrated a halo of spared fat.

\section{Discussion}

$\mathrm{SM}$ is most common in white men over the age of $50^{5}$ as our patient. In systematic review of 192 cases done by Sharma et all almost $60 \%$ of patients were while males with the mean age of 55years with a male to female ratio of 2.3:1. ${ }^{6}$ Similarly, in a review of 92cases $70 \%$ were males and the median age group was 66years. ${ }^{5}$ One study mentioned that men and women are equally affected without any racial predominance 1 . Children are not as commonly affected which may be because they have less fat than adults. ${ }^{5}$

Sclerosing Mesenteritis is characterized by fat degeneration, necrosis, chronic inflammation, scarring and eventually fibrosis of the fatty tissue within the mesentery. ${ }^{7}$ The most commonly affected are the mesentery of the small bowel and the mesoappendix even though the large bowel mesentery is less commonly affected. ${ }^{8}$ The exact mechanism is unknown; however, several conditions have been associated with SM. ${ }^{5}$ The study by Sharma et all on 192cases showed that $28.6 \%$ of the cases had history of abdominal trauma or prior surgery, $8.9 \%$ had history of cancer and $5.7 \%$ had underlying autoimmune disease. ${ }^{6}$ Some authors have implicated the association of SM with IgG4 related disease. ${ }^{1}$ IgG4 related disease which is a fibroinflammatory condition is characterized by formation of tumor like growth in multiple sites of the body and elevated IgG4 level in the serum. ${ }^{9}$ Kim et al., ${ }^{10}$ reported a SM case in a 45 years old woman and Minato et al., ${ }^{11}$ reported a SM case in a 53years old man both of which concurrently had IgG related disease. ${ }^{10,11}$ Other studies have shown the association of SM with carcinomas like breast, Colo-rectal, gynecological and urological cancers and hematological malignancies like Non-Hodgkin's Lymphoma and Multiple Myeloma. ${ }^{1,9-17}$ In a review of 359 cases with SM like CT findings, $27.9 \%$ of the cases had either new or old cancer. Lymphoma was the most common malignancy followed by prostate, melanoma, lung and renal cell cancer. ${ }^{18,19}$ Furthermore; studies have shown association of SM with conditions like sclerosing cholangitis, Riedel thyroiditis, mixed connective tissue disease, ankylosing spondylitis, Sjogren's syndrome tuberculosis, acute pancreatitis and granulomatous diseases. ${ }^{1,13,20}$ Only

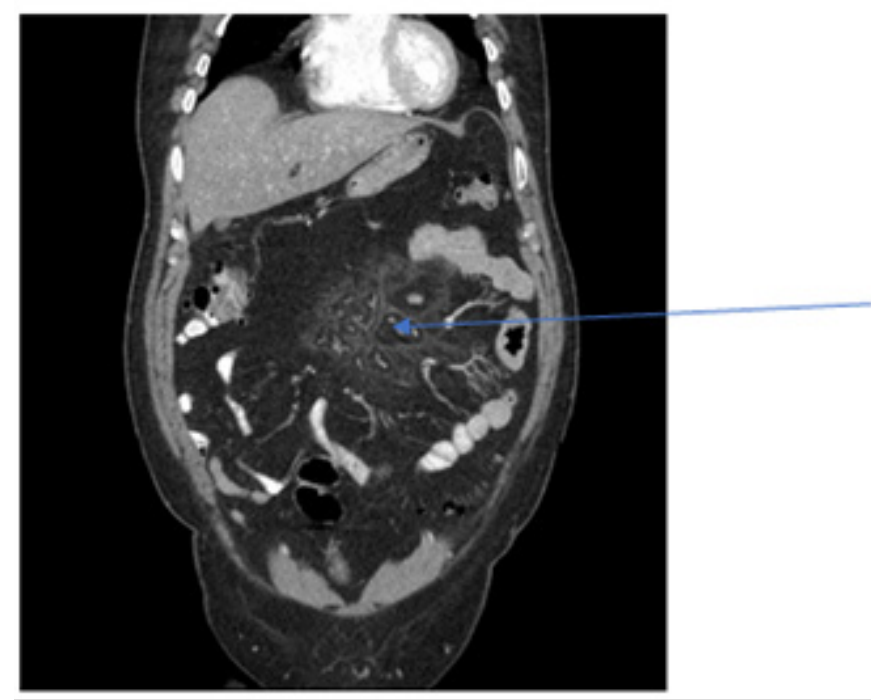

Figure 3 Repeat CT abdomen/pelvis one year later showed stable appearance of inflamed fat at the root of mesentery with numerous small lymph nodes.

significant finding in our patient was partially incarcerated hernia.

Patients with SM may be asymptomatic or present with symptoms. ${ }^{1}$ The study by Sharma and Akram showed that the most common presenting symptom was chronic abdominal pain followed by fever. Other symptoms included weight loss, diarrhea, vomiting, anorexia, constipation, bloating, malaise, nausea, pain with eating and fatigue. ${ }^{6}$ $\mathrm{He} \mathrm{H}$ et al., ${ }^{7}$ reported a 77years old male who presented with dyschezia, abdominal pain and mass in left lower quadrant and Katsuya Endo et all reported a 22years old Japanese male who presented with chronic abdominal pain and small intestine mesentery mass. ${ }^{7,21}$ Harvin G reported a case of SM who presented with intestinal obstruction. ${ }^{22}$ On physical examination majority of the patients have a normal abdominal exam however others may elicit abdominal tenderness, abdominal mass and ascites based on the underlying cause. ${ }^{5,6}$ Our patient presented with chronic right lower quadrant abdominal pain with partially incarcerated abdominal hernia.

The laboratory findings of SM are non-specific. ${ }^{7}$ Majority of the patients have elevated C-reactive protein (CRP) and erythrocyte sedimentation rate (ESR). The study by Sharma et all showed that $86.5 \%$ of patients had elevated CRP and $88.4 \%$ of the cases had elevated ESR. Other nonspecific laboratory findings include leukocytosis, anemia and low total protein levels. ${ }^{6}$ Those with concurrent IgG4 related disease will have elevated IgG4 levels in the blood. ${ }^{9,11}$ CT scan has been useful in diagnosing SM however it is not diagnostic. ${ }^{23}$ Owing to the rarity of the cases, the CT findings are described as "Hazy" or "misty" mesentery or unexplained mesenteric mass. ${ }^{19}$ The usual CT scan findings are mesenteric lymphadenopathy, fat sparing the mesenteric vessels also known are fat sparing sign, hazy mesenteric fat and separation of mesenteric vessels, increased thickness of the involved mesentery, and well or poorly defined mesenteric mass, pseudo capsule (soft-tissue surrounding the inflamed mass). ${ }^{4,23}$ These are usually described as a mass in the mesentery with fat enhancement with multiple soft tissue nodes along the root of the mesentery without vascular compromise. ${ }^{24}$ Due to the involvement of the jejunal mesentery, the mass is usually found in the left side. ${ }^{23}$ MRI 
is not usually performed for the diagnosis of SM, however if done, it is characterized by hyper intense signal in the $\mathrm{T} 2 \mathrm{~W}$ fat sat indicating edema and intermediate signal density with multiple strands due to fibrotic retraction of the mesentery on T1W signals. When fibrosis is prevalent it manifests as delayed hyper enhancement on $\mathrm{T} 2 \mathrm{~W}$ images. ${ }^{23}$ On pathology grossly, there is thickening of the mesentery which can be diffuse, single knotty of multiple knotty. ${ }^{1}$ Histologically there is a mixture of fat necrosis, chronic inflammation and fibrosis which involve the mesenteric and submucosal fat of the bowel and the mucosa remains intact. ${ }^{1}$ In almost half of the vases the most common finding is extensive fibrosis with inflammation and little fat necrosis while almost one quarter of the cases have predominant chronic inflammation and other quarter has lipid-laden foamy macrophages. ${ }^{5}$ Our patient had CT scan findings suggestive of SM which was confirmed by gross and histologic findings in pathology.

Due to the rarity of the condition, there are no specific guidelines for treatment of SM however steroids, colchicine, azathioprine, thalidomide, cyclophosphamide and progesterone have been tired, however asymptomatic cases don't need therapy. Surgical resection is recommendation for non-resolving cases and complicated by bowel obstruction. ${ }^{1,25}$ Sharma et al reported that out of 192 cases, $41.7 \%$ of cases received surgical resection, $34.9 \%$ underwent medical treatment and $17.2 \%$ cases were conservatively managed. Of those who received medical $83.5 \%$ got steroids and $11.9 \%$ received colchicine, tamoxifen and 6-mercaptopurine each. Remainder of the patients either received 6-mercaptopurine, antibiotics, azathioprine, methotrexate and infliximab. Other rare agents used were cyclophosphamide, IVIG, dipenicillamine and tacrolimus. Of the medical treatment receivers, $78.6 \%$ had complete resolution of symptoms and $20.8 \%$ patients had complications the common being bowel ischemia, obstructive uropathy/renal failure, steroid related complications, sepsis/respiratory failure and venous thromboembolism. Rarer complications included pericardial effusion and pulmonary edema. The overall all-cause mortality rate was $7.3 \%$ and the common causes of death were postoperative complications, bowel ischemia, sepsis and bronchopneumonia, venous thromboembolism, renal failure, venous thromboembolism, renal failure, cardiac arrest and pulmonary edema. At 10 months follow up more than half the patients had abdominal tenderness. ${ }^{6}$ A treatment algorithm was prepared by Akram after review on 92cases. Asymptomatic patients with incidental diagnosis do not need treatment. Patients with features of bowel obstruction need to undergo surgical treatment followed by tamoxifen plus tapering dose of steroids if there is persistence of symptoms. First line treatment for symptomatic patients not requiring surgery including tamoxifen with tapering doses of prednisone. For patients who are contraindicated for tamoxifen especially due to thromboembolism should be treated with colchicine, azathioprine or thalidomide. ${ }^{5}$ Of the 92 cases about $73 \%$ of the patients were followed up within a month. Out of these, $26 \%$ patients remained asymptomatic and $74 \%$ continued to have mild abdominal symptoms. ${ }^{5}$ Our patient had surgery for repair of the hernia but not all the affected mesenteries were removed. Several months after the diagnosis, he had a comfortable life with on and off mild abdominal pain which was resolved by ibuprofen.

Finally, SM is a rare care of chronic abdominal pain. Though most of the cases are benign like ours, they can be associated with underlying malignancy which need to be ruled out. There are no specific guidelines for treatment of SM till date. Further studies are required to formulate specific guidelines for the treatment of SM for which our case could add value.

\section{Acknowledgements}

None.

\section{Disclaimer}

\section{Author's contribution}

None.

\section{Conception and design}

Santosh Raj Sharma, Denzil Etienne.

\section{Drafting of the article}

Santosh Raj Sharma, Denzil Etienne, Dhuha Alhankawi, Jaafar Al-dahwi

\section{Critical revision of the article for important intellectual content}

Santosh Raj Sharma, Denzil Etienne, Dhuha Alhankawi, Jaafar Aldahwi, Madhavi Reddy.

\section{Final approval of the article}

Santosh Sharma, Denzil Etienne, Dhuha Alhankawi, Jaafar Aldahwi, Madhavi Reddy

\section{Consent for publication}

Informed consent was obtained from the patient.

\section{Funding}

This work received no specific grant from any funding agency in the public, commercial, or not-for-profit sectors.

\section{Conflict of interest}

The authors have no conflict of interest or financial relationships to disclose.

\section{References}

1. Hussein MR, Abdelwahed SR. Mesenteric panniculitis: an update. Expert Rev Gastroenterol Hepatol. 2015;9(1):67-78.

2. Emory TS, Monihan JM, Carr NJ, et al. Sclerosing mesenteritis, mesenteric panniculitis and mesenteric lipodystrophy: a single entity? Am J Surg Pathol. 1997;21(4):392-398.

3. Halligan S, Plumb A, Taylor S. Mesenteric panniculitis: systematic review of cross-sectional imaging findings and risk of subsequent malignancy. Eur Radiol. 2016;26(12):4531-4537.

4. Daskalogiannaki M, Voloudaki A, Prassopoulos P, et al. CT evaluation of mesenteric panniculitis: prevalence and associated diseases. AJR Am J Roentgenol. 2000;174(2):427-431.

5. Akram S, Pardi DS, Schaffner JA, et al. Sclerosing mesenteritis: clinical features, treatment, and outcome in ninety-two patients. Clin Gastroenterol Hepatol. 2007;5(5):523-584.

6. Sharma P, Yadav S, Needham CM, et al. Sclerosing mesenteritis: a 
systematic review of 192 cases. Clin J Gastroenterol. 2017;10(2):103111.

7. He H, Zhi M, Zhang M, et al. Sclerosing Mesenteritis: Multidisciplinary collaboration is essential for diagnosis and treatment. Gastroenterology Res. 2017;10(1):50-55.

8. Popkharitov AI, Chomov GN. Mesenteric panniculitis of the sigmoid colon: a case report and review of the literature. J Med Case Rep. 2007;1:108

9. Deshpande V, Zen Y, Chan JK, et al. Consensus statement on the pathology of IgG4-related disease. Mod Pathol. 2012;25(9):1181-1192.

10. Kim EJ, Kim EY, Song JE, et al. A case of IgG4-related sclerosing mesenteritis associated with Crohn's disease. Korean J Gastroenterol. 2014;63(3):176-182.

11. Minato H, Shimizu J, Arano Y, et al. IgG4-related sclerosing mesenteritis: a rare mesenteric disease of unknown etiology. Pathol Int. 2012;62(4):281286.

12. Bechade D, Durand X, Desrame J, et al. Etiologic spectrum of mesenteric panniculitis: report of 7 cases. Rev Med Interne. 2007;28(5):289-295.

13. Goh J, Otridge B, Brady H, et al. Aggressive multiple myeloma presenting as mesenteric panniculitis. Am J Gastroenterol. 2001;96(1):238-241.

14. Guerri Fernandez R, Villar Garcia J, Garcia Sirvent L, et al. Mesenteric panniculitis, first clinical manifestation of a disseminated B cell lymphoma. Rev Clin Esp. 2008;208(2):109-110.

15. Harris RJ, van Stolk RU, Church JM, et al. Thoracic mesothelioma associated with abdominal mesenteric panniculitis. Am J Gastroenterol. 1994;89(12):2240-2242.
16. Okamoto M, Ohkubo N, Yamanaka M, et al. [A case of mesenteric panniculitis complicated with malignant lymphoma]. Nihon Shokakibyo Gakkai Zasshi. 1998;95(8):884-889.

17. Wilkes A, Griffin N, Dixon L, et al. Mesenteric panniculitis: a paraneoplastic phenomenon? Dis Colon Rectum. 2012;55(7):806-809.

18. Buchwald P, Diesing L, Dixon L, et al. Cohort study of mesenteric panniculitis and its relationship to malignancy. $\mathrm{Br} J$ Surg. 2016;103(12):1727-1730.

19. Ehrenpreis ED, Roginsky G, Gore RM. Clinical significance of mesenteric panniculitis-like abnormalities on abdominal computerized tomography in patients with malignant neoplasms. World J Gastroenterol. 2016;22(48):10601-10608.

20. Mohamed H, Mehdi C, Ikram M, et al. Mesenteric panniculitis associated with acute pancreatitis: about a case. Pan Afr Med J. 2016;24:206.

21. Endo K, Moroi R, Sugimura M, et al. Refractory sclerosing mesenteritis involving the small intestinal mesentery: a case report and literature review. Intern Med. 2014;53(13):1419-1427.

22. Harvin G, Graham A. Sclerosing mesenteritis: a rare cause of small bowel obstruction. Case Rep Gastroenterol. 2016;10(1):63-67.

23. Apostolakis S, Ioannidis A, Tsioga G, et al. A systematic investigation of sclerosing mesenteritis through CT and MRI. Radiol Case Rep. 2016;11(4):299-302.

24. Scheer F, Spunar P, Wiggermann P, et al. Mesenteric panniculitis (MP) in CT-a predictor of malignancy? Rofo. 2016;188(10):926-932.

25. Klasen J, Guller U, Muff B, et al. Treatment options for spontaneous and postoperative sclerosing mesenteritis. World $J$ Gastrointest Surg. 2016;8(11):761-765. 